\title{
Endometrial Atypical Hyperplasia with Unusually Prominent Apoptosis: A Case Report
}

\section{Elizabeth Arslanian*, M. Ruhul Quddus, Kamaljeet Singh}

Department of Pathology, Brown University / Women \& Infants Hospital, Providence, Rhode Island, 101 Dudley Street, Providence, RI 02905, USA

*Corresponding Author: Elizabeth Arslanian, Department of Pathology, Brown University / Women \& Infants Hospital, Providence, Rhode Island, 101 Dudley Street, Providence, RI 02905, USA

Received date: 13 November 2021; Accepted date: 20 November 2021; Published date: 27 November 2021

Citation: Arslanian E, Quddus MR, Singh K (2021) Endometrial Atypical Hyperplasia with Unusually Prominent Apoptosis: A Case Report. J Med Case Rep Case Series 2(14): https://doi.org/10.38207/JMCRCS/2021/0214219

Copyright: (C) 2021 Elizabeth Arslanian. This is an open-access article distributed under the terms of the Creative Commons Attribution License, which permits unrestricted use, distribution, and reproduction in any medium, provided the original author and source are credited.

\begin{abstract}
A 67-year-old patient presented with uterine bleeding and a thickened endometrium on imaging. The endometrial biopsy revealed glandular crowding with cytologic atypia in a background of proliferative endometrium, overall consistent with endometrial atypical hyperplasia. Abundant apoptotic bodies populated the neoplastic glands, which is unusual for an endometrioid phenotype. The immunostaining for Bcl-2, an anti-apoptotic marker, was reduced in the neoplastic glands as compared to the background endometrium. The diagnosis of endometrial atypical hyperplasia was confirmed on the hysterectomy specimen. Prominent apoptosis in endometrial tissue is more common in the benign process of endometrial breakdown and tumors like serous carcinoma and HPV-associated endocervical adenocarcinoma. Prominent apoptosis is an unusual histologic finding in endometrioid carcinoma and its precursor endometrial atypical hyperplasia. Usually, there is the diminished activity of the apoptosis regulator FOXO1 in endometrioid carcinoma. Our case is an exception to this rule, showing that extensive apoptosis can also be seen in endometrial atypical hyperplasia.
\end{abstract}

Keywords: Apoptosis; Endometrial Hyperplasia; EIN; Carcinoma, Endometrioid; Endometrium; Hysterectomy; Curettage

\section{*Graphical abstract description}

A 67-year-old patient presented with uterine bleeding. Her endometrial biopsy represented in this image (H\&E, 20X) was heterogeneous appearing with crowding of complex glands with cytologic atypia. Of note, abundant apoptotic bodies were noted in the neoplastic glands, with diminished Bcl-2 immunostaining. The

\section{Case report}

A 67-year-old G1P1 woman presented with uterine bleeding and stated that she had never undergone menopause and had never been on hormone replacement therapy. Her past medical history was otherwise significant for menarche at ten years old and morbid

\section{Discussion and Conclusion}

Histological examination revealed abundant endometrial tissue. A low-power magnification view revealed a heterogeneous pattern with areas in which glands exceeded stroma in volume in a background of proliferative endometrium. A complex architectural pattern was present in the hyperplastic glands with a scant stromal component Neoplastic cells were distinct from the background endometrium, and some showed secretory change. Neoplastic cells were atypical with mildly enlarged rounder nuclei and small nucleoli. A notable finding was the abundance of apoptotic bodies without a background of stromal or glandular breakdown. Focal foamy histiocytes were noted background endometrium (not shown) was proliferative. The findings were consistent with endometrial atypical hyperplasia / endometrial intraepithelial neoplasia, with the unusual morphologic feature of extensive apoptosis.

obesity. A CT scan showed a thickened endometrium. An endometrial biopsy showed an atypical glandular proliferation with abundant apoptotic bodies.

(Figure 1). Immunostaining revealed strong nuclear expression of progesterone receptor (PR), increased $\mathrm{Ki}-67$ proliferation index (up to $80 \%$ of nuclei in affected glands), wild-type p53 pattern, and patchy p16. Bcl-2 immunostaining showed reduced intensity in the neoplastic cells compared to strong cytoplasmic staining in the background endometrium (Figure 2). We rendered a diagnosis of endometrial atypical hyperplasia. The microscopic examination of the endometrium from hysterectomy confirmed the diagnosis of endometrial atypical hyperplasia without associated carcinoma. 


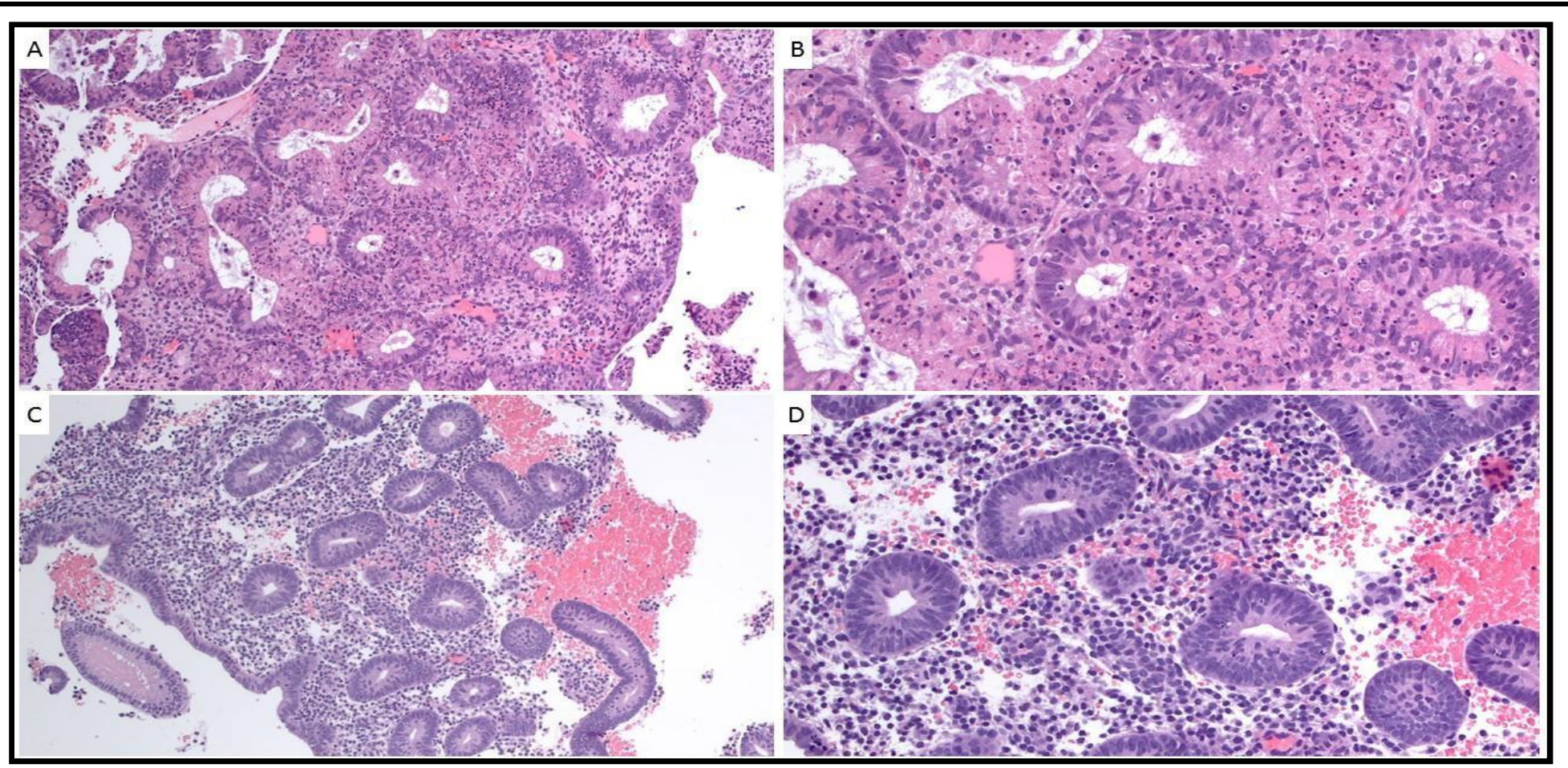

Figure 1: H\&E. A (10X) and B (20X): Endometrial atypical hyperplasia: glandular crowding with scant residual stroma between the glands, cytologic atypia and abundant apoptotic bodies. C (10X) and D (20X): Background proliferative endometrium: spaced out tubular and round mitotically active glands with pseudostratified nuclei associated with abundant stroma.

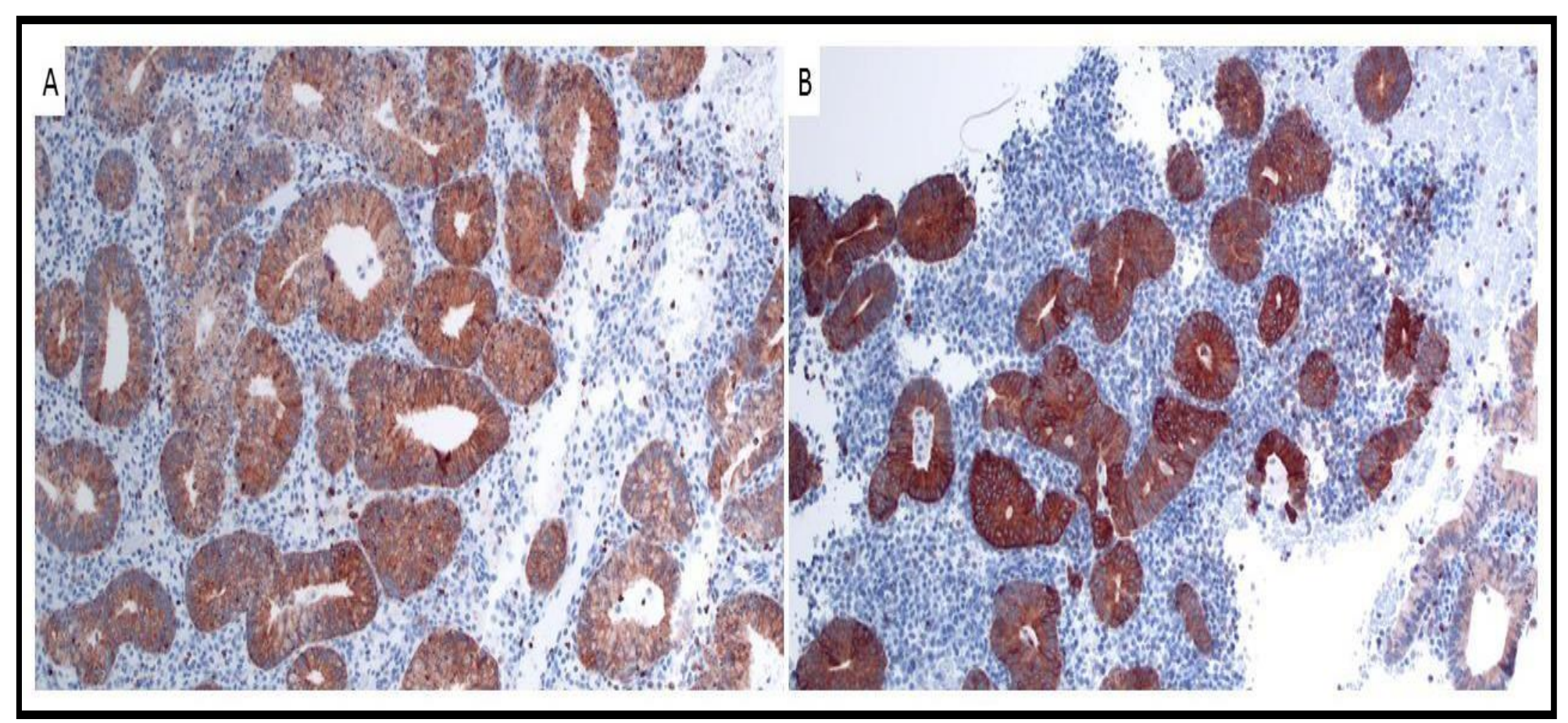

Figure 2: Bcl-2 immunostaining, 10X. A: Endometrial atypical hyperplasia: diffuse low to moderate cytoplasmic staining in glandular neoplastic cells. B: Background proliferative endometrium: diffuse strong cytoplasmic staining in glandular non-neoplastic cells.

In an endometrial sample, the presence of malignant glands with abundant apoptotic bodies indicates type II (non-endometrioid) endometrial carcinoma, or an HPV-associated endocervical adenocarcinoma. Extensive apoptosis is an infrequent morphologic finding in type I (endometrioid) endometrial carcinoma and there is even evidence of reduced apoptotic activity in endometrioid endometrial carcinoma. The pro-apoptotic FOXO1 transcription factor is expressed in non-neoplastic FOXO1 endometrial glandular cells. Alterations in FOXO1 activity have been shown in a study by Goto et al. FOXO1 transcripts were more abundant in non-neoplastic endometrium than in endometrioid endometrial carcinoma. FOXO1 immunostaining was strong in benign endometrial epithelial cells. It was negative or weak in endometrioid carcinoma samples. [1] In vitro initiation of apoptosis in endometrial carcinoma cells can be obtained through induced overexpression of FOXO1. [2] As previously mentioned, extensive apoptosis as a morphologic finding is more common in HPV-associated endocervical adenocarcinoma and in type II (non-endometrioid) carcinoma. It is one of the hallmarks features used to distinguish HPV-associated endocervical adenocarcinoma from endometrial endometrioid carcinoma. Other criteria in favor of an endocervical primary include apical mitoses, nuclear pleomorphism, increased mitotic activity, and associated adenocarcinoma in-situ (AIS). Past or present endometrial atypical hyperplasia or endometrial intraepithelial neoplasia (EIN), squamous metaplasia, and stromal foamy histiocytes are most consistent with endometrial carcinoma. An immunohistochemistry panel consisting of PR, ER, p16, vimentin, and mCEA provides further clues into the tumor origin. Positive PR, ER, and vimentin stains favor an endometrial primary, whereas mCEA expression and block-positive p16 block tip the scale to favor an endocervical origin. Also, in situ hybridization detection of high-risk HPV is expected to be positive for most cases of HPV-associated cervical adenocarcinoma. [3] Clinical correlation also proves beneficial. Endometrial endometrioid carcinoma occurs mainly in the setting of postmenopausal bleeding. HPV-associated cervical adenocarcinoma primarily affects a younger population. 
In our case, a 67-year-old woman presented with uterine bleeding, which raised a concern for endometrial neoplasia. Her endometrial biopsy consisted of crowded but not confluent atypical endometrial glands, distinct from her background proliferative endometrium. The presence of abundant apoptotic bodies was unusual. As per 2020 WHO classification of female genital tumors, endometrial atypical hyperplasia $(\mathrm{EAH})$ / endometrioid intraepithelial neoplasia (EIN) refers to areas of crowded cytologically atypical glands that are morphologically distinct from the background endometrium. Immunohistochemistry studies are desirable but not required for diagnosis. These include mismatch repair proteins, PAX2 and PTEN, which can all show loss of expression in EAH/EIN. The recognition of $\mathrm{EAH} / \mathrm{EIN}$ is of paramount importance as up to one-third of cases

Funding sources: No funding sources were involved in this work.

Conflicts of interest: The authors declare no conflicts of interest.

Acknowledgments: Not applicable

\section{References}

1. Goto T, Takano M, Albergaria A, Briese J, Pomeranz KM, et al. (2008) Mechanism and functional consequences of loss of FOXO1 expression in endometrioid endometrial cancer cells. Oncogene. 27: 9-19.

2. Berry E, Hardt JL, Clardy J, Lurain JR, Kim JJ (2009) Induction of apoptosis in endometrial cancer cells by psammaplysene A involves FOXO1. Gynecol Oncol. 112(2): 331-6. are associated with carcinoma at surgery performed within the same year. [4]

All in all, although the presence of extensive apoptosis is typically a feature of stromal and glandular breakdown, HPV-associated endocervical adenocarcinoma and type II (non-endometrioid) endometrial carcinoma, it can also be seen in endometrial atypical hyperplasia. This case illustrates the adage that anything is possible in medicine "The disease does not read textbooks".

Consent: Written informed consent was obtained from the patient for publication of their case.

Authors' contribution: EA, MRQ, and KS are involved in paper preparation.

3. Ronnett BM (2016) Endocervical adenocarcinoma: selected diagnostic challenges. Mod Pathol. 29 Suppl 1: S12-28.

4. WHO Classification of Tumours Editorial Board. Female genital tumours. Lyon (France): International Agency for Research on Cancer; 2020. (WHO classification of tumours series, $5^{\text {th }}$ ed.; vol.4). 